\title{
Patient Orientation Column
}

National Cancer Institute

\section{Source}

National Cancer Institute. Patient Orientation Column. NCI Thesaurus. Code C69205.

The orientation of the patient relative to the image plane designated in the anatomical direction of the column axis. 